\title{
Detailed analysis of the performance of the modified Becke-Johnson potential
}

\author{
J. A. Camargo-Martínez and R. Baquero \\ Departamento de Física, CINVESTAV-IPN, Av. IPN 2508, 07360 México
}

(Dated: October 30, 2018)

\begin{abstract}
Very recently, in the 2011 version of the Wien2K code, the long standing shortcome of the codes based on Density Functional Theory, namely, its impossibility to account for the experimental band gap value of semiconductors, was overcome. The novelty is the introduction of a new exchange and correlation potential, the modified Becke-Johnson potential (mBJLDA). In this paper, we report our detailed analysis of this recent work. We calculated using this code, the band structure of forty one semiconductors and found an important improvement in the overall agreement with experiment as Tran and Blaha [Phys. Rev. Lett. 102, 226401 (2009)] did before for a more reduced set of semiconductors. We find, nevertheless, within this enhanced set, that the deviation from the experimental gap value can reach even much more than $20 \%$, in some cases. Furthermore, since there is no exchange and correlation energy term from which the mBJLDA potential can be deduced, a direct optimization procedure to get the lattice parameter in a consistent way is not possible as in the usual theory. These authors suggest that a LDA or a GGA optimization procedure is used previous to a band structure calculation and the resulting lattice parameter introduced into the 2011 code. This choice is important since small percentage differences in the lattice parameter can give rise to quite higher percentage deviations from experiment in the predicted band gap value. We found that by using the average of the two lattice parameters (LDA and GGA) a better agreement with the band gap experimental value is systematically obtained. As a rule, the LDA optimization underestimates the lattice parameter while the GGA one overestimates it. Also we found that using the experimental lattice parameter instead, surprisingly high deviations of the predicted band gap value from experiment, occur. This is an odd result since, in general, the quality of the LDA and GGA obtained lattice parameters are judged to be as good as their proximity to the experimental lattice parameter value. This judgment implies the idea that the best result for the predicted band gap value is obtained when the closest-to-experiment lattice parameter is used. On the other hand, the band structure calculated with the mBJLDA potential seems, at first sight, a simple rigid displacement of the conduction bands towards higher energies. A closer look reveals that, in some cases, important differences occur that might not be negligible in certain systems containing a semiconductor as it might happen at interfaces. So, in some systems containing a semiconductor, neither the direct use of the Wien $2 \mathrm{k}$ previous version nor its use with a rigid displacement of the conduction bands added so as to reproduce the band gap value, are totally reliable. The overall implementation of the calculation of the band structure of semiconductors with the Wien2k code using this new potential is quite empirical although it mimics well the results obtained by other methods as the GW approximation which give better results and are theoretically well founded. We conclude that, in spite of the very important improvement in the band gap agreement with experiment using the mBJLDA potential, there are issues that point to the fact that this problem is not yet totally closed.
\end{abstract}




\section{INTRODUCTION}

Wien2k [1] has been one of the most used codes to calculate the band structure of solids since long ago. In 2011, the long standing shortcome, i.e., its impossibility to reproduce the experimental gap of semiconductors, has been approximately overcome in the Wien2k 2011 version 2]. This code is based on the Full Potential (Linearized) augmented plane waves and local orbitals [FP-(L)APW+lo] [3] where a new exchange and correlation potential was introduced. The result is a very remarkable improvement with respect to the previous version. Some cases worth noting remain as an exception but the overall agreement is good. While computing the gap of a certain number of semiconductors (41), we found that optimization plays a role since small differences in the lattice parameter can give, for the same semiconductor, noticeable differences in the gap. It was observed that small changes in the lattice parameter between $0.02 \%$ and $1.0 \%$, can induce differences between $0.5 \%$ and $6.5 \%$ (see text below). The optimization procedure gives the value of the lattice parameter that is consistent with the minimum of the energy vs. volume curve. As such, it depends on the interchange and correlation energy, $E_{x c}$, used in the calculation. In principle, it appears that the consistent procedure should be to use in the optimization procedure from which the lattice parameter is obtained, the exchange and correlation energy functional from which the exchange and correlation potential to be used in the electronic band structure is deduced. The previous version of the Wien2K code uses either LDA or GGA in this way but as it is well known, neither formulation reproduces accurately enough the gap of semiconductors. The exchange and correlation potential, the modified Becke-Johnson potential (mBJLDA), does a much better job. Blaha et al. 2] got the best results when the lattice parameter is first obtained from a LDA or a GGA optimization followed by a band structure calculation using the new mBJLDA exchange and correlation potential. This way of performing the calculation is necessary since it is impossible to obtain an expression for a functional $E_{x c}$ such that the mBJLDA potential is $V_{m B J}=\delta E_{x c}[\rho] / \delta \rho$, as in the usual theory. In this work, we analyse the results obtained with the mBJLDA to pinpoint to its assets and to its odds in more detail. We conclude that even though the overall agreement with experiment of the band gap value obtained with the Wien2k 2011 constitutes a noticeable improvement, there are some cases and details that point to the fact that the problem might not be really totally closed.

\section{DENSITY FUNCTIONAL THEORY}

Several codes were developed based on Density Functional Theory (DFT) which became the most used, precise and practical way to calculate the band structure of solids. The development of practical approximations to the exchange and correlation energy functional lead to a remarkable degree of accuracy to describe even complicated metallic systems. At the basis of DFT is the Hohenberg-Khon theorem [4] which shows that the knowledge of the density of the ground state is equivalent to the one of the wave function itself. The density of states of the real ground state many body system is equal to the one calculated from the solution of the Khon-Sham equations [5],

$$
\left[T+V_{H}+V_{x c}+V_{e x t}\right] \varphi_{i}(r)=\varepsilon_{i} \varphi_{i}
$$

where the density is calculated taking into account the occupied states only. In eq.(1), $T$ is the kinetic energy operator, $V_{H}$ is the Hartree potential, $V_{e x t}$ is the external potential and $V_{x c}$ is the exchange and correlation potential which is calculated from the exchange and correlation energy functional; $V_{x c}=\delta E_{x c}[\rho] / \delta \rho$. To solve the Khon-Sham equations (1), an explicit expression for $E_{x c}[\rho]$ is needed. The exact expression is unknown since it includes all kind of correlations between all the particles in the system. So an approximation is needed. The first and best known approximation is the Local Density Approximation, LDA [6], which was followed by the Generalized Gradient Approximation (GGA) 7] and the meta-GGA [7] among others. These potentials reproduce rather well the band structure of even complicated metallic systems but fail to reproduce the gap of semiconductors. As a possible empirical solution to this problem, Blaha et al. 2] have reported the mBJLDA potential which is a modification of the exchange and correlation potential of Becke and Johnson (BJ) [8]. The new potential reproduces the experimental gap of semiconductors with accuracy several orders of magnitude better than the previous version of the Wien2K code using either the LDA or the GGA. The mBJLDA potential is

$$
V_{x, \sigma}^{M B J}(r)=c V_{x, \sigma}^{B R}(r)+(3 c-2) \frac{1}{\pi} \sqrt{\frac{5}{12}} \sqrt{\frac{2 t_{\sigma}(r)}{\rho_{\sigma}(r)}}
$$

Where $\rho_{\sigma}(r)$ is the spin dependent density of states, $t_{\sigma}(r)$ is the kinetic energy density and $V_{x, \sigma}^{B J}(r)$ is the Becke-Roussel potential $(B R)[9]$. The c stands for

$$
c=\alpha+\beta\left(\frac{1}{V_{\text {cell }}} \int d^{3} r \frac{|\nabla \rho(r)|}{\rho(r)}\right)^{1 / 2}
$$


$\alpha$ and $\beta$ are free parameters. The Wien2k 2011 code defines $\alpha=-0.012$ and $\beta=1.023 B o h r^{1 / 2}$. These values are general but certainly fixed experimenting with several cases. No expression for the exchange and correlation energy is given and therefore no total energy functional is really possible. It is in this sense that the formulation might be seen as an empirical model in spite of its universality. We next explore further some more details of it.

\section{THE OPTIMIZATION PROCEDURE}

The optimization procedure in the usual theory, uses the proposed energy functional to get the structural groundstate parameters of the solid at $\mathrm{T}=0 \mathrm{~K}$. These parameters are then used in a further step to get the band structure in a consisted way. It is in this sence that the exact way used for the optimization procedure is important. The Wien2k code allows the calculation of the equilibrium structural properties of the system, the minimum of the total energy, $\left(E_{0}\right)$, the Bulk modulus, $\left(B_{0}\right)$, its derivative with respect to pressure, $\left(B_{0}^{\prime}\right)$, and the equilibrium volume at zero pressure, $V_{0}$, by fitting the data to an equation of state (EOS). The code uses either the EOS by Murnaghan [10] or the one by Birch-Murnaghan [11] or else the so-called EOS2 [12]. We used the first one,

$$
\begin{gathered}
E(V)=E_{0}+\frac{B_{0} V}{B_{0}^{\prime}}\left[\frac{1}{\left(B_{0}^{\prime}-1\right)}\left(\frac{V_{0}}{V}\right)^{B_{0}^{\prime}}+1\right]-\frac{B_{0} V_{0}}{\left(B_{0}^{\prime}-1\right)} \\
P(V)=\frac{B_{0}}{B_{0}^{\prime}}\left[\left(\frac{V_{0}}{V}\right)^{B_{0}^{\prime}}-1\right]
\end{gathered}
$$

and performed the calculation for some semiconductors ( $\mathrm{Si}$, Ge, AlAs, $\mathrm{SiC}, \mathrm{BP}, \mathrm{AlP}, \mathrm{GaN}, \mathrm{GaAs}, \mathrm{LiF}, \mathrm{MgO}$ and $\mathrm{BN}$ ), using the LDA and GGA approximations.

TABLE I. The Bulk modulus, $\left(B_{0}\right)$, in GPa and its derivative, $\left(B_{0}^{\prime}\right)$, as obtained from the Murghanan fit. The crystal structure is shown in parenthesis. Experimental values were taken from references [13 23].

\begin{tabular}{lcccccc}
\hline \hline & \multicolumn{2}{c}{ LDA } & \multicolumn{2}{c}{ GGA } & \multicolumn{2}{c}{ Expt. } \\
\hline Solid & $B_{0}$ & $B_{0}^{\prime}$ & $B_{0}$ & $B_{0}^{\prime}$ & $B_{0}$ & $B_{0}^{\prime}$ \\
\hline C(A1) & 434.34 & 3.69 & 432.52 & 3.99 & 442 & 4.03 \\
Si(A1) & 92.96 & 4.35 & 87.71 & 4.23 & 97.82 & 4.09 \\
Ge(A1) & 73.18 & 4.98 & 62.45 & 3.93 & 75.80 & 4.55 \\
MgO(B1) & 175.52 & 4.61 & 149.89 & 4.18 & 160.00 & 4.15 \\
LiF(B3) & 69.29 & 4.91 & 68.07 & 4.22 & 62.00 & 5.14 \\
AlAs(B3) & 73.34 & 4.78 & 67.29 & 4.53 & 78.10 & - \\
SiC(B3) & 233.85 & 4.06 & 214.95 & 4.11 & 224.00 & 4.1 \\
BP(B3) & 175.12 & 3.85 & 160.91 & 3.84 & 173.00 & 3.76 \\
AlP(B3) & 89.70 & 4.17 & 82.15 & 3.97 & 86.00 & 3.99 \\
BN(B3) & 401.27 & 3.71 & 370.10 & 3.66 & 369.00 & 4.00 \\
GaN(B3) & 202.45 & 3.28 & 177.39 & 4.11 & 188.00 & 3.2 \\
GaAs(B3) & 75.15 & 3.97 & 67.14 & 4.03 & 74.66 & 4.6 \\
\hline \hline
\end{tabular}

In the Table \ we present these results first for the Bulk modulus $\left(B_{0}\right)$ and its derivative with respect to pressure $\left(B_{0}^{\prime}\right)$. We can see that when calculated with the LDA, the deviations from experiment of the Bulk modulus and its derivative oscillate roughly between 1-12\%; with the GGA between $4-18 \%$. The values for the Bulk modulus as compared to experiment are overestimated in all cases but three ( $\mathrm{Si}, \mathrm{Ge}, \mathrm{AlAs}$ ) when an LDA optimization is used. For a GGA one, all are underestimated but two ( $\mathrm{LiF}, \mathrm{BN})$. The GGA optimization gives a better agreement with experiment in five of the eleven semiconductors considered ( $\mathrm{MgO}, \mathrm{LiF}, \mathrm{AlP}, \mathrm{BN}, \mathrm{GaN})$. Looking at the derivative of the Bulk modulus with respect to pressure (seven experimental results reported), the GGA optimization gives a better agreement with experiment in four cases ( $\mathrm{Si}, \mathrm{Ge}, \mathrm{MgO}, \mathrm{GaN}$ ).

One the problem with the mBJLDA potential is that since no exchange and correlation energy functional, $E_{x c}$, is defined within this formulation, no consistent optimization procedure is possible. This shortcome is the result of the empirical character of the mBJLDA potential. As a somehow empirical and inconsistent solution to this shortcome, Tran and Blaha 2] suggest to start with an GGA (or LDA) optimization and to introduce the lattice parameter value obtained into the band structure calculation code that uses the mBJLDA potential. 
TABLE II. Lattice parameters, $(a)$, obtained from the Murnaghan fit. The $a_{A v g}$ values are the average value between $a_{L D A}$ and $a_{G G A}$. The experimental data, at low temperature (LT) and room temperature (RT), are from refs. 23 [25].

\begin{tabular}{|c|c|c|c|c|c|c|}
\hline \multirow[b]{2}{*}{ Solid } & \multicolumn{6}{|c|}{ Lattice parameters $a[\AA]$} \\
\hline & & $a_{L D A}$ & $a_{G G A}$ & $a_{A v g}$ & $a_{L T}^{E x p t}$ & $a_{R T}^{\text {Expt. }}$ \\
\hline $\mathrm{C}(\mathrm{A} 1)$ & & 3.5339 & 3.5731 & 3.5535 & $3.5667^{*}$ & 3.5668 \\
\hline $\operatorname{Si}(\mathrm{A} 1)$ & & 5.4073 & 5.4738 & 5.4406 & $5.4298^{*}$ & 5.4310 \\
\hline $\mathrm{Ge}(\mathrm{A} 1)$ & & 5.6269 & 5.7586 & 5.6928 & $5.6524^{*}$ & 5.6579 \\
\hline $\mathrm{MgO}(\mathrm{B} 1)$ & & 4.1635 & 4.2569 & 4.2102 & $4.2052^{* \mathrm{a}}$ & 4.2110 \\
\hline $\mathrm{LiF}(\mathrm{B} 3)$ & & 3.9152 & 4.0710 & 3.9931 & - & 4.0300 \\
\hline $\operatorname{AlAs}(\mathrm{B} 3)$ & & 5.6329 & 5.7304 & 5.6817 & $5.6605^{*}$ & 5.6614 \\
\hline $\mathrm{SiC}(\mathrm{B} 3)$ & & 4.3378 & 4.3944 & 4.3661 & $4.3585_{3 \mathrm{~K}}^{\mathrm{b}}$ & 4.3596 \\
\hline BP(B3) & & 4.4937 & 4.5510 & 4.5224 & - & 4.5383 \\
\hline $\mathrm{AlP}(\mathrm{B} 3)$ & & 5.4371 & 5.5110 & 5.4741 & - & 5.4635 \\
\hline BN(B3) & & 3.5831 & 3.6268 & 3.6050 & - & 3.6155 \\
\hline GaN(B3) & & 4.4663 & 4.5626 & 4.5145 & - & 4.5230 \\
\hline GaAs(B3) & & 5.6050 & 5.7401 & 5.6726 & $5.6523^{*}$ & 5.6533 \\
\hline BAs(B3) & & 4.7314 & 4.8085 & 4.7700 & - & 4.777 \\
\hline $\operatorname{InP}(\mathrm{B} 3)$ & & 5.8239 & 5.9535 & 5.8887 & $5.8657_{4.8 \mathrm{~K}}$ & 5.87875 \\
\hline AlSb(B3) & & 6.1084 & 6.2209 & 6.1647 & - & 6.1355 \\
\hline GaSb(B3) & & 6.0449 & 6.2086 & 6.1268 & - & 6.09593 \\
\hline GaP(B3) & & 5.3912 & 5.5084 & 5.4498 & $5.4469^{*}$ & 5.4508 \\
\hline InAs(B3) & & 6.0185 & 6.1804 & 6.0995 & - & 6.0584 \\
\hline $\operatorname{InSb}(\mathrm{B} 3)$ & & 6.4497 & 6.6363 & 6.5430 & - & 6.4794 \\
\hline CdS(B3) & & 5.7636 & 5.9304 & 5.8470 & - & 5.8320 \\
\hline $\mathrm{CdTe}(\mathrm{B} 3)$ & & 6.4166 & 6.6198 & 6.5182 & $6.4675^{\mathrm{c}}$ & 6.4810 \\
\hline CdSe(B3) & & 6.0169 & 6.1995 & 6.1082 & - & 6.0770 \\
\hline ZnS(B3) & & 5.3083 & 5.4524 & 5.3804 & - & 5.4102 \\
\hline ZnSe(B3) & & 5.5826 & 5.7401 & 5.6614 & - & 5.6680 \\
\hline ZnTe(B3) & & 6.0129 & 6.1854 & 6.0992 & - & 6.0890 \\
\hline $\operatorname{MgS}(\mathrm{B} 1)$ & & 5.1358 & 5.2342 & 5.1850 & - & 5.2030 \\
\hline MgS(B3) & & 5.5985 & 5.7018 & 5.6502 & - & 5.6220 \\
\hline $\operatorname{MgSe}(\mathrm{B} 1)$ & & 5.3993 & 5.5129 & 5.4561 & - & 5.4600 \\
\hline $\operatorname{MgTe}(\mathrm{B} 3)$ & & 6.3836 & 6.5187 & 6.4512 & - & 6.4230 \\
\hline $\mathrm{BaS}(\mathrm{B} 1)$ & & 6.2743 & 6.4313 & 6.3528 & - & $6.3890^{\mathrm{d}}$ \\
\hline $\operatorname{BaSe}(\mathrm{B} 1)$ & & 6.4792 & 6.6604 & 6.5698 & - & $6.5950^{\mathrm{d}}$ \\
\hline $\operatorname{BaTe}(\mathrm{B} 1)$ & & 6.8647 & 7.0680 & 6.9664 & - & $7.0070^{\mathrm{d}}$ \\
\hline $\mathrm{CaO}(\mathrm{B} 1)$ & & 4.7177 & 4.8346 & 4.7762 & - & 4.8110 \\
\hline $\mathrm{CuCl}$ (B3) & & 5.2101 & 5.4403 & 5.3252 & $5.4093^{* a}$ & 5.4202 \\
\hline $\mathrm{CuBr}(\mathrm{B} 3)$ & & 5.5264 & 5.7391 & 5.6328 & $5.6764^{*}$ & 5.6900 \\
\hline $\mathrm{AgF}(\mathrm{B} 1)$ & & 4.7946 & 5.0224 & 4.9085 & - & 4.9360 \\
\hline $\operatorname{AgI}(B 1)$ & & 6.3656 & 6.6416 & 6.5036 & - & 6.4990 \\
\hline \multirow[t]{2}{*}{$\mathrm{GaN}(\mathrm{B} 4)$} & $\mathrm{a}$ & 3.2027 & 3.2051 & 3.2039 & - & 3.1980 \\
\hline & $\mathrm{c}$ & 5.1408 & 5.1330 & 5.1369 & - & 5.1850 \\
\hline \multirow[t]{2}{*}{$\operatorname{InN}(\mathrm{B} 4)$} & $\mathrm{a}$ & 3.5715 & 3.5751 & 3.5733 & - & 3.5480 \\
\hline & $\mathrm{c}$ & 5.6845 & 5.6729 & 5.6787 & - & 5.7600 \\
\hline \multirow[t]{2}{*}{$\operatorname{AlN}(\mathrm{B} 4)$} & a & 3.1200 & 3.1211 & 3.1206 & $3.1113^{* \mathrm{e}}$ & 3.1120 \\
\hline & c & 4.9565 & 4.9529 & 4.9547 & $4.9793^{* \mathrm{e}}$ & 4.9820 \\
\hline \multirow[t]{2}{*}{$\mathrm{ZnO}(\mathrm{B} 4)$} & a & 3.2675 & 3.2604 & 3.2640 & $3.2482_{4.2 \mathrm{~K}}^{\mathrm{f}}$ & 3.2500 \\
\hline & $\mathrm{c}$ & 5.1736 & 5.1653 & 5.1695 & $5.2040_{4.2 \mathrm{~K}}^{\mathrm{f}}$ & 5.2070 \\
\hline
\end{tabular}

*Extrapolated values. ${ }^{\mathrm{a}}$ Ref. [26], ${ }^{\mathrm{b}}$ Ref. [27],

${ }^{\mathrm{c}}$ Ref. [28], ${ }^{\mathrm{d}}$ Ref. [29], ${ }^{\mathrm{e}}$ Ref. [30], ${ }^{\mathrm{f}}$ Ref. [31]. 
In Table I. we present the lattice parameter, $a$, calculated with LDA, GGA, its average value, $a_{A v g},\left(a_{A v g}=\right.$ $\left.\left(a_{L D A}+a_{G G A}\right) / 2\right)$ and the experimental reports at low temperature (LT) and at room temperature (RT). Notice that the LDA gives rise to deviations of the lattice parameter that are always below the experimental value (except for the values of $a$ in hexagonal structures) while exactly the contrary arises with the GGA (except for the values of $c$ in hexagonal structures). GGA results in a shift towards higher values of the equilibrium volume which might be thought as a kind of relaxation due to the use of derivatives of the local density in the functional. The experimental lattice parameters were taken from refs. 23 25]. In Table I] we first compare the lattice parameter obtained with an LDA optimization to experimental data taken at low temperature (LT) and at room temperature (RT). In the first case, we find, in general, small differences (0.6-1\%), except for $\mathrm{CuBr}$ and $\mathrm{CuCl}$ (2.6 and 3.7, respectively). In the second case, the differences are usually below $1.3 \%$ but can rise up to $3.9 \%$ as in $\mathrm{CuCl}$. If we use the GGA approximation the differences are slightly higher in general.

We will show below that the use of the average lattice parameter, $a_{A v g}$, results in a gap value that is always in better agreement with experiment. We give an error statistics analysis of this result in Table III to emphasise its validity. To obtain $a_{A v g}$ has a low computational extra cost.

TABLE III. Lattice parameter error statistics for compounds of Table【in in

\begin{tabular}{|c|c|c|c|}
\hline \multicolumn{4}{|c|}{ Error relative to LT experiments. } \\
\hline & $a_{L D A}$ & $a_{G G A}$ & $a_{A v g}$ \\
\hline $\mathrm{ME}^{\mathrm{a}}$ & -0.046 & 0.047 & 0.00041 \\
\hline $\mathrm{MAE}^{\mathrm{b}}$ & 0.050 & 0.055 & 0.025 \\
\hline $\mathrm{SD}^{\mathrm{c}}$ & 0.046 & 0.049 & 0.034 \\
\hline \multicolumn{4}{|c|}{ Error relative to RT experiments. } \\
\hline & $a_{L D A}$ & $a_{G G A}$ & $a_{A v g}$ \\
\hline $\mathrm{ME}^{\mathrm{a}}$ & -0.055 & 0.055 & -0.00009 \\
\hline $\mathrm{MAE}^{\mathrm{b}}$ & 0.058 & 0.066 & 0.026 \\
\hline $\mathrm{SD}^{\mathrm{c}}$ & 0.048 & 0.055 & 0.034 \\
\hline
\end{tabular}

${ }^{a}$ Mean error. ${ }^{\mathrm{b}}$ Mean absolute error.

${ }^{\mathrm{c}}$ Standard deviation.

\section{BAND STRUCTURE CALCULATIONS}

In Fig 1 we compare the band structure for Si, Ge, GaAs and LiF calculated with the LDA and with the mBJLDA potential. As a general result, this potential causes a rigid displacement of the conduction bands toward higher energies with respect to the top of the valence band with small differences in the dispersion at some regions of the Brillouin zone but reproducing, in general, the characteristic behavior of the bands for each semiconductor according to experiment [23, 24, 32, 33] as the Wien2k code used to. If we look at the resulting band structures in more detail, we see that a rigid displacement of only the conduction bands as to reproduce the mBJLDA predicted gap value will cause some differences in the upper conduction bands although the ones just above the upper gap edge will match quite well with the calculation using the mBJLDA potential. This is roughly true for the first three semiconductors presented in Fig 1. LiF deserves a more detailed discussion. In Fig 2, we show the result of such a displacement. We can see that clear differences arise that might influence a calculation of a system containing LiF as one of its elements. So, we conclude that, in general, a rigid displacement of a band structure calculation with the old formulation of Wien2K will not be accurate enough for certain purposes as it might occur in the calculation of the band structure of certain interfaces containing a semiconductor as one of its elements, for example. The direct use of the previous version of the Wien2K code in this case, will not be accurate enough as well.

In Table IV we show the result of the calculation using the mBJLDA potential with the $a_{L D A}, a_{G G A}$ and $a_{A v g}$ to survey the respective differences in the gap value as compared to experiment [23 25]. We can see that if we use the lattice parameter from a LDA optimization, $\mathrm{mBJ}\left(a_{L D A}\right)$, the prediction for the gap deviates by less than $10 \%$ for 21 of the 41 semiconductors considered, between 10-20\% for 9 of them and more then $20 \%$ for the remaining 11 (Ge and GaAs among them). Using GGA, we get 19, 14, and 6, respectively. When using the average value of the two, we get 25, 7, and 7 a result that improves the calculation at relatively low computational cost. There are, nevertheless, two semiconductors that we did not include in the two last cases just presented, MgS(B3) and MgTe. 
Si(A1)

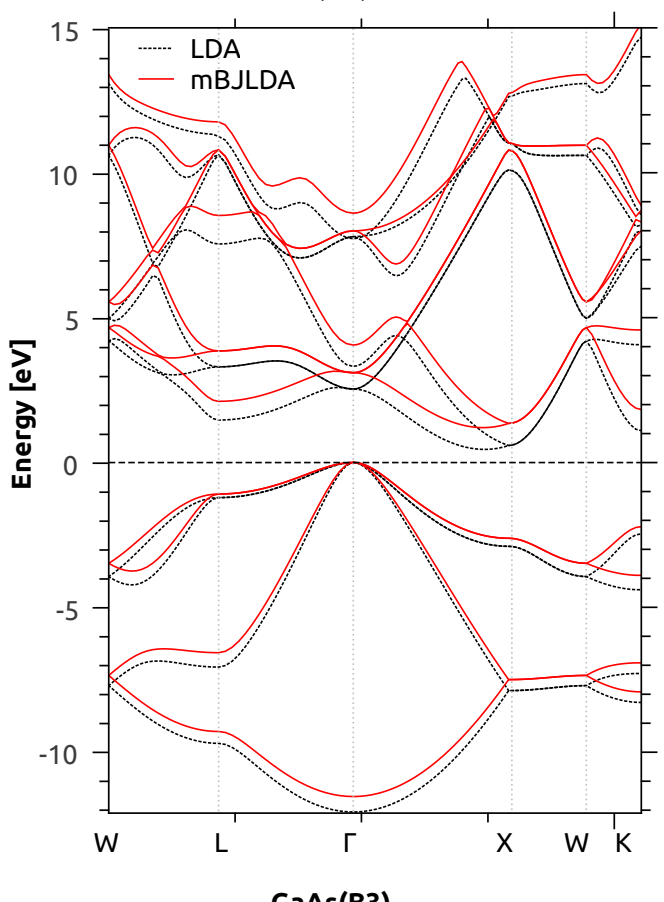

$\operatorname{GaAs(B3)}$

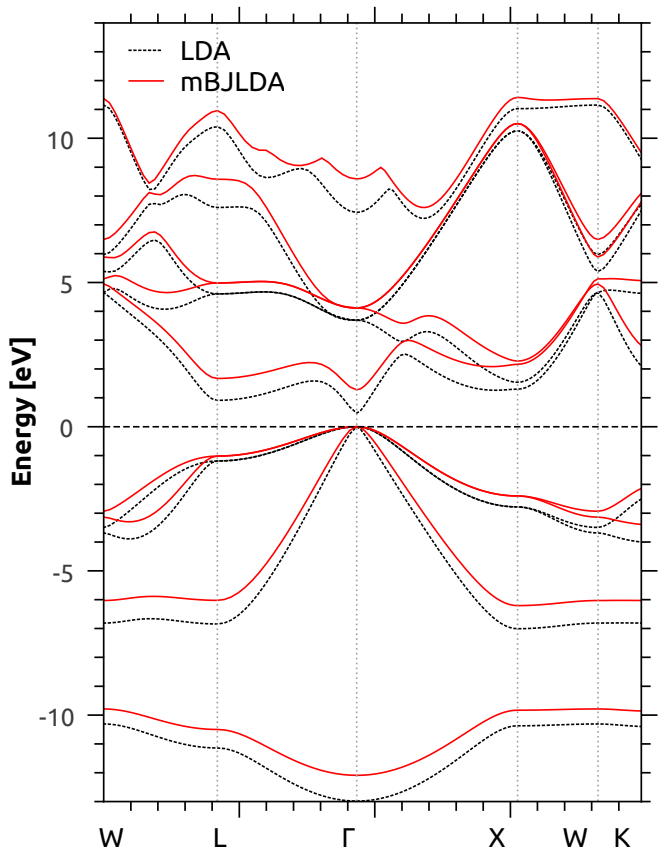

Ge(A1)
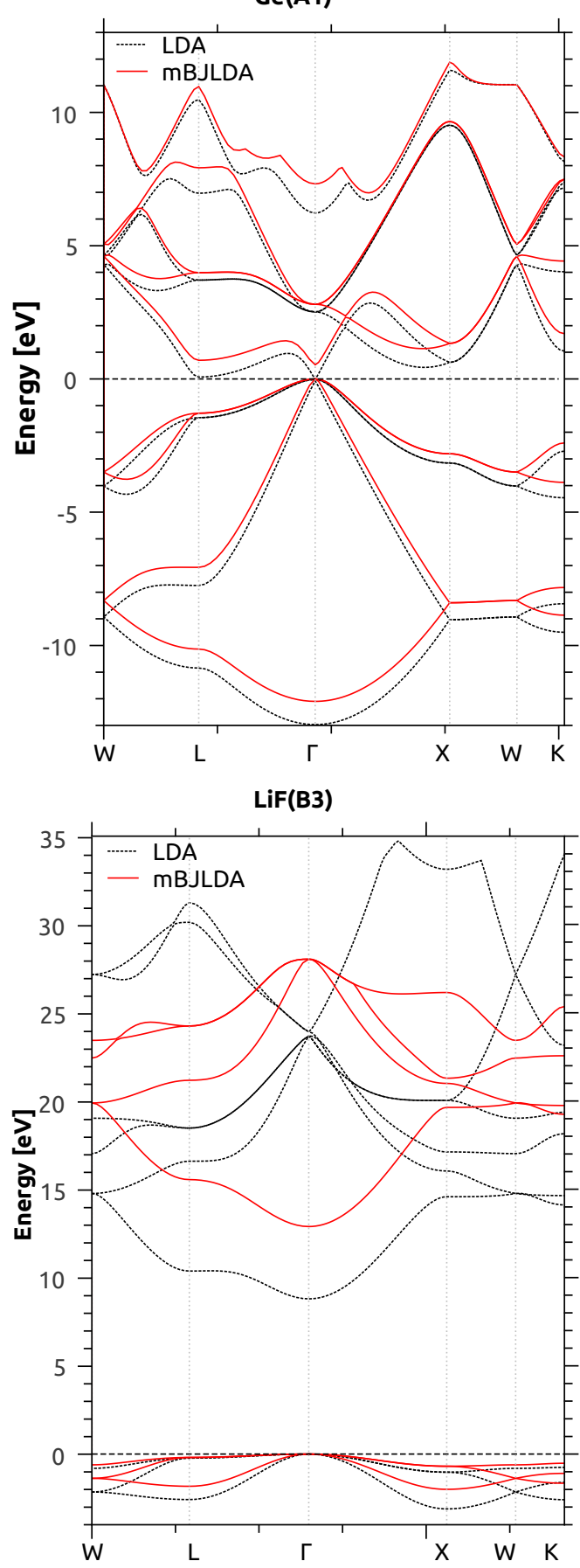

FIG. 1. The band structure obtained for Si, Ge, GaAs, and LiF with LDA and mBJLDA. The origin is at the top of the valence band. In parenthesis the crystal structure is shown.

It turns out that both, the calculation using $a_{G G A}$ and $a_{A v g}$ give the wrong result that the gap is indirect while $a_{L D A}$ gives rise to a correct direct gap for both semiconductors and predicts its value with less than $10 \%$ deviation in both cases. Further, to explore the absolute capacity of the mBJLDA potential to predict the gap value correctly, we have used the experimental lattice parameter value at low temperature $\left(a_{L T}\right)$ where it existed in the literature to perform the calculation. It is interesting that the result is not as good as one would expect as it can be seen from Table IV. This calculation shows the best that the mBJLDA potential can do, a result that might be important to bear in mind. It looks like that when no data are available, the best result is obtained by taking the average of the 


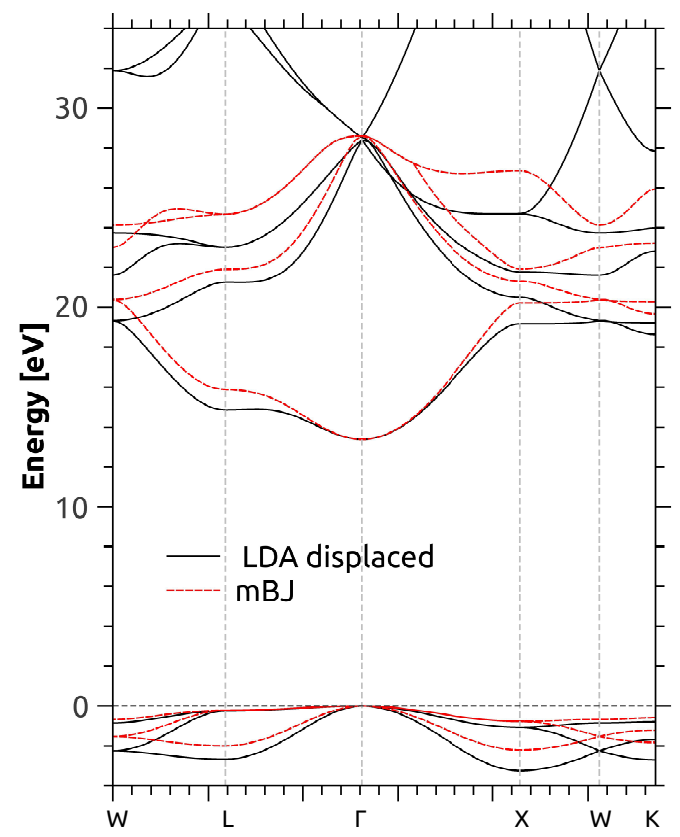

FIG. 2. The band structure obtained for LiF with LDA and mBJLDA.

lattice parameters resulting from an LDA optimization and a GGA one. Furthermore, it is interesting to observe that small differences in the lattice parameter can give rise to noticeable differences in the predicted value for the gap. An example of this is Ge. A small difference in the lattice parameter as $0.5 \%$ (LDA) gives rise to a $23 \%$ deviation of the gap value as compared to experiment. Within the GGA optimization, a $1.9 \%$ lattice difference generates a $5.4 \%$ off value for the gap and a $0.6 \%$ difference in the lattice parameter gives an $8.1 \%$ difference in the gap when the average is taken. A very interesting issue is that when the experimental value of the lattice parameter is introduced into the mBJLDA code, important deviations from experiment occur. In Table IV] we have included 14 semiconductors for which we found an experimental low temperature lattice parameter value. We get the experimental gap value only for one of them $(\mathrm{Si})$. With less than $10 \%$ deviation we found 7 (C, MgO, AlAs, $\mathrm{SiC}, \mathrm{GaAs}, \mathrm{GaP}$, and $\mathrm{CdTe}$ ); a deviation between 10-20\% occurs in 3 (Ge, InP, and $\mathrm{AlN})$. Very important deviations from experiment occur in $\mathrm{CuCl}$ ( $47.5 \%)$, and $\mathrm{CuBr}(45.3 \%)$. These results are facts to bear in mind for a proper evaluation of the performance of the mBJLDA potential since the quality of the optimization procedure is judged from the deviation of the predicted lattice parameter from the experimental value. This judgment implies that the best result for the predicted band gap value is obtained when the experimental lattice parameter is introduced into the code. Actually any code should, first of all, be examined in this sense so that the selection of an optimization procedure based on the deviation of the predicted lattice parameter from experiment, really makes sense. So, the mBJLDA potential gives rise to inconsistencies that emphasise its empirical character. 
TABLE IV. Calculations of the gap $\left(E_{g}\right)$ in eV, from the potential mBJLDA using the parameters in Table II The lattice parameter $a_{A v g}$ is the average value between $a_{L D A}$ and $a_{G G A}$. The experimental values were taken from references [23 25]. The crystal structure and percentage difference with respect to experiment is shown in parenthesis.

\begin{tabular}{|c|c|c|c|c|c|}
\hline \multirow[b]{2}{*}{ Solid } & \multicolumn{5}{|c|}{ Gap } \\
\hline & $E_{g}^{m B J\left(a_{L D A}\right)}$ & $E_{g}^{m B J\left(a_{G G A}\right)}$ & $E_{g}^{m B J\left(a_{L T}\right)}$ & $E_{g}^{m B J\left(a_{A v g}\right)}$ & $E_{g}^{E x p t}$ \\
\hline$\overline{C(A 1)}$ & $4.98(-9.1 \%)$ & $4.92(-10.2 \%)$ & $4.93(-10.0 \%)$ & $4.95(-9.7 \%)$ & $5.48 *$ \\
\hline $\mathrm{Si}(\mathrm{A} 1)$ & $1.13(-3.4 \%)$ & $1.20(2.6 \%)$ & $1.17(0.0 \%)$ & $1.17(0.0 \%)$ & $1.17^{*}$ \\
\hline $\mathrm{Ge}(\mathrm{A} 1)$ & $0.91(23 \%)$ & $0.70(-5.4 \%)$ & $0.84(13.5 \%)$ & $0.80(8.1 \%)$ & $0.74_{1.5 \mathrm{~K}}$ \\
\hline $\mathrm{MgO}(\mathrm{B} 1)$ & $7.57(-2.6 \%)$ & $6.88(-11.5 \%)$ & $7.26(-6.6 \%)$ & $7.22(-7.1 \%)$ & $7.77_{7.7 \mathrm{~K}}$ \\
\hline $\operatorname{AlAs}(\mathrm{B} 3)$ & $2.13(-4.5 \%)$ & $2.22(-0.4 \%)$ & $2.14(-4.0 \%)$ & $2.17(-2.7 \%)$ & $2.23_{4 \mathrm{~K}}$ \\
\hline $\mathrm{SiC}(\mathrm{B} 3)$ & $2.21(-8.7 \%)$ & $2.28(-5.8 \%)$ & $2.26(-6.6 \%)$ & $2.26(-6.6 \%)$ & $2.42_{2 \mathrm{~K}}$ \\
\hline $\mathrm{AlP}(\mathrm{B} 3)$ & $2.28(-6.9 \%)$ & $2.37(-3.3 \%)$ & - & $2.33(-4.9 \%)$ & $2.45_{4 \mathrm{~K}}$ \\
\hline GaN(B3) & $3.13(-10.6 \%)$ & $2.74(-21.7 \%)$ & - & $2.94(-16.0 \%)$ & $3.50_{1.6 \mathrm{~K}}$ \\
\hline GaAs(B3) & $1.84(21.1 \%)$ & $1.28(-15.8 \%)$ & $1.62(6.6 \%)$ & $1.56(2.6 \%)$ & $1.52^{*}$ \\
\hline $\operatorname{InP}(\mathrm{B} 3)$ & $1.70(19.7 \%)$ & $1.34(-5.6 \%)$ & $1.59(12.0 \%)$ & $1.52(7.0 \%)$ & $1.42_{1.6 \mathrm{~K}}$ \\
\hline $\mathrm{AlSb}(\mathrm{B} 3)$ & $1.76(4.8 \%)$ & $1.84(9.5 \%)$ & - & $1.80(7.1 \%)$ & $1.68_{27 \mathrm{~K}}$ \\
\hline GaSb(B3) & $1.20(46.3 \%)$ & $0.68(-17.1 \%)$ & - & $0.90(9.8 \%)$ & $0.82^{*}$ \\
\hline GaP(B3) & $2.18(-7.2 \%)$ & $2.30(-2.1 \%)$ & $2.24(-4.7 \%)$ & $2.24(-4.7 \%)$ & $2.35^{*}$ \\
\hline InAs(B3) & $0.77(83.3 \%)$ & $0.34(-19.0 \%)$ & - & $0.55(31.0 \%)$ & $0.42_{4.2 \mathrm{~K}}$ \\
\hline InSb(B3) & $0.59(145.8 \%)$ & $0.09(-62.5 \%)$ & - & $0.31(29.2 \%)$ & $0.24_{1.8 \mathrm{~K}}$ \\
\hline CdS(B3) & $2.68(8.1 \%)$ & $2.52(1.6 \%)$ & - & $2.61(5.2 \%)$ & $2.48_{4.2 \mathrm{~K}}$ \\
\hline $\mathrm{CdTe}(\mathrm{B} 3)$ & $1.80(12.5 \%)$ & $1.54(-3.8 \%)$ & $1.73(8.1 \%)$ & $1.67(4.4 \%)$ & $1.60^{*}$ \\
\hline CdSe(B3) & $1.99(12.4 \%)$ & $1.77(0.0 \%)$ & - & $1.87(5.6 \%)$ & $1.77^{*}$ \\
\hline $\mathrm{ZnS}(\mathrm{B} 3)$ & $3.85(1.0 \%)$ & $3.55(-6.8 \%)$ & - & $3.70(-2.9 \%)$ & $3.81^{*}$ \\
\hline ZnSe(B3) & $2.90(2.8 \%)$ & $2.56(-9.2 \%)$ & - & $2.74(-2.8 \%)$ & $2.82_{6 \mathrm{~K}}$ \\
\hline ZnTe(B3) & $2.52(5.4 \%)$ & $2.19(-8.4 \%)$ & - & $2.38(-0.4 \%)$ & $2.39_{2 \mathrm{~K}}$ \\
\hline $\operatorname{MgS}(\mathrm{B} 1)$ & $4.12(-8.4 \%)$ & $4.02(-10.7 \%)$ & - & $4.07(-9.6 \%)$ & $4.50_{77 \mathrm{~K}}$ \\
\hline $\operatorname{MgS}(\mathrm{B} 3)$ & $5.18(-4.1 \%)$ & - & - & - & $5.40_{5 \mathrm{~K}}^{\mathrm{a}}$ \\
\hline $\operatorname{MgTe}(\mathrm{B} 3)$ & $3.59(-2.2 \%)$ & - & - & - & $3.67_{2 \mathrm{~K}}^{\mathrm{b}}$ \\
\hline $\mathrm{CaO}(\mathrm{B} 1)$ & $7.55(-3.2 \%)$ & $6.97(-10.6 \%)$ & - & $7.31(-6.3 \%)$ & $7.80^{*+}$ \\
\hline $\mathrm{CuCl}(\mathrm{B} 3)$ & $2.00(-41 \%)$ & $1.72(-49.3 \%)$ & $1.78(-47.5 \%)$ & $1.85(-49.3 \%)$ & $3.39_{2 \mathrm{~K}}$ \\
\hline $\mathrm{CuBr}(\mathrm{B} 3)$ & $1.83(-40.4 \%)$ & $1.62(-47.2 \%)$ & $1.68(-45.3 \%)$ & $1.71(-44.3 \%)$ & $3.07_{1.6 \mathrm{~K}}$ \\
\hline $\operatorname{AgF}(\mathrm{B} 1)$ & $1.80(-35.7 \%)$ & $2.44(-12.9 \%)$ & - & $2.22(-20.7 \%)$ & $2.80_{4.8 \mathrm{~K}}$ \\
\hline $\operatorname{AgI}(\mathrm{B} 1)$ & $2.72(-6.5 \%)$ & $2.83(-2.7 \%)$ & - & $2.77(-4.8 \%)$ & $2.91_{4 \mathrm{~K}}$ \\
\hline $\mathrm{GaN}(\mathrm{B} 4)$ & $3.13(-5.2 \%)$ & $3.13(-5.2 \%)$ & - & $3.13(-5.2 \%)$ & $3.30_{10 \mathrm{~K}}^{\mathrm{c}}$ \\
\hline $\operatorname{InN}(\mathrm{B} 4)$ & $0.82(15.5 \%)$ & $0.82(15.5 \%)$ & - & $0.82(15.5 \%)$ & $0.71_{10 \mathrm{~K}}^{\mathrm{d}}$ \\
\hline $\operatorname{AlN}(B 4)$ & $5.52(-10.8 \%)$ & $5.53(-10.7 \%)$ & $5.56(-10.2 \%)$ & $5.53(-10.7 \%)$ & $6.19_{7 \mathrm{~K}}^{\mathrm{e}}$ \\
\hline $\mathrm{ZnO}(\mathrm{B} 4)$ & $2.77(-19.5 \%)$ & $2.77(-19.5 \%)$ & $2.72(-20.9 \%)$ & $2.76(-19.7 \%)$ & $3.44^{*}$ \\
\hline $\mathrm{LiF}(\mathrm{B} 1)$ & $13.80(1.5 \%)$ & $12.92(-5.0 \%)$ & - & $13.40(-1.5 \%)$ & $13.6_{300 \mathrm{~K}}^{\mathrm{f}}$ \\
\hline BP(B3) & $1.80(-10.0 \%)$ & $1.87(-6.5 \%)$ & - & $1.83(-8.5 \%)$ & $2.00_{300 \mathrm{~K}}$ \\
\hline $\mathrm{BN}(\mathrm{B} 3)$ & $5.86(-5.5 \%)$ & $5.83(-6.0 \%)$ & - & $5.85(-5.6 \%)$ & $6.20_{300 \mathrm{~K}}$ \\
\hline $\operatorname{MgSe}(\mathrm{B} 1)$ & $2.95(19.4 \%)$ & $2.84(15.0 \%)$ & - & $2.89(17.0 \%)$ & $2.47_{300 \mathrm{~K}}$ \\
\hline $\mathrm{BaS}(\mathrm{B} 1)$ & $3.23(-16.8 \%)$ & $3.37(-13.1 \%)$ & - & $3.31(-14.7 \%)$ & $3.88_{300 \mathrm{~K}}$ \\
\hline $\mathrm{BaSe}(\mathrm{B} 1)$ & $2.80(21.8 \%)$ & $2.94(-17.9 \%)$ & - & $2.87(-19.8 \%)$ & $3.58_{300 \mathrm{~K}}$ \\
\hline $\mathrm{BaTe}(\mathrm{B} 1)$ & $2.13(-30.8 \%)$ & $2.34(-24.0 \%)$ & - & $2.24(-27.3 \%)$ & $3.08_{300 \mathrm{~K}}$ \\
\hline $\mathrm{BAs}(\mathrm{B} 3)$ & $1.69(15.8 \%)$ & $1.75(19.9 \%)$ & - & $1.72(17.8 \%)$ & $1.46_{300 \mathrm{~K}}$ \\
\hline
\end{tabular}

${ }^{*}$ Extrapolated values. ${ }^{+}$Reported value are direct gap at $\Gamma .{ }^{a} \operatorname{Ref}[34] .{ }^{b}$ Ref. [35].

${ }^{\mathrm{d}} \operatorname{Ref}[36] .{ }^{\mathrm{e}} \operatorname{Ref}[37] .{ }^{\mathrm{f}} \operatorname{Ref}$ [38]. ${ }^{\mathrm{c}} \operatorname{Ref}$. [39]. 
TABLE V. Band gap error statistics for compounds in Tables IV in eV. (See text)

\begin{tabular}{|c|c|c|c|c|}
\hline \multicolumn{5}{|c|}{ Error relative to LT experiments. } \\
\hline & $\operatorname{mBJ}\left(a_{L D A}\right)$ & $\operatorname{mBJ}\left(a_{G G A}\right)$ & $\operatorname{mBJ}\left(a_{A v g}\right)$ & $\operatorname{mBJ}\left(a_{L T}\right)$ \\
\hline $\mathrm{ME}^{\mathrm{a}}$ & -0.15 & -0.32 & -0.24 & -0.87 \\
\hline $\mathrm{MAE}^{\mathrm{b}}$ & 0.33 & 0.35 & 0.30 & 0.45 \\
\hline $\mathrm{SD}^{\mathrm{c}}$ & 0.44 & 0.43 & 0.42 & 1.99 \\
\hline \multicolumn{5}{|c|}{ Error relative to RT experiments. } \\
\hline & $\mathrm{mBJ}(\mathrm{LDA})$ & mBJ(GGA) & $\mathrm{mBJ}\left(a_{\text {Avg }}\right)$ & \\
\hline $\mathrm{ME}^{\mathrm{a}}$ & -0.25 & -0.30 & -0.27 & \\
\hline $\mathrm{MAE}^{\mathrm{b}}$ & 0.48 & 0.47 & 0.44 & \\
\hline
\end{tabular}

${ }^{\mathrm{a}}$ Mean error. ${ }^{\mathrm{b}}$ Mean absolute error.

${ }^{\mathrm{c}}$ Standard deviation.

We finally present in TableV, the statistical analysis of the gap value obtained from a band structure calculation with the mBJLDA potential using as input different lattice parameters obtained from different optimization procedures. The use of the average lattice parameter gives clearly the best result. It is worth noting that when the experimental parameter at low temperature, $\mathrm{mBJ}\left(a_{L T}\right)$, is introduced in the code to calculate the band gap values, we get, in some cases, deviation from the experimental value of the gap that are not expected, in principle.

In a further work [40] we compare the predicted bsnd gap value as obtained by different methods in the literature. The theoretically well found GW approximation gives rise to the best predictions. Nevertheless the mBJLDA empirical potential results compare acceptable well.

\section{CONCLUSIONS}

We have calculated using the empirical mBJLDA potential 2], the band structure of forty one semiconductors and got their band gap value which we compared to experiment. In this formulation, there is no expression for the exchange and correlation energy so that the mBJLDA potential can be obtained from it, as in the usual theory. Due to this shortcome, no consistent optimization procedure is possible. This is due to the empirical character of the mBJLDA potential. As an empirical solution to this problem, Tran and Blaha [2] suggest the use of a LDA or a GGA optimization to get the lattice parameter that goes in a further step into the code that calculates the band structure using the mBJLDA potential. As an overall first sight picture, the mBJLDA potential causes a rigid displacement of the conduction band towards higher energies so as to considerably improve the agreement with experiment of the band gap values. A closer look reveals that, in some cases, there are noticeable deviations from a rigid displacement of the band structure calculated with the previous version of the code that might result in wrong conclusions as it could happen when the band structure of an interface is calculated, for example. We explored at this point the resulting band gap predicted value as a function of the lattice parameter used. We found two important facts. First, the best result for the band gap value is obtained, in general, if the average lattice parameter, $a_{A v g}$, is used $\left(a_{A v g}=\left(a_{L D A}+a_{G G A}\right) / 2\right)$ where $a_{L D A}\left(a_{G G A}\right)$ is the lattice parameter that results from a LDA(GGA) optimization. Second, another important result is that if we take the experimental lattice parameter into the code using the mBJLDA potential, important deviations from the experimental gap value are obtained, a result to bear in mind for a detailed analysis of the performance of the mBJLDA potential. This result is important since it shades the optimization procedure in the sense that a lattice parameter closer to experiment does not guarantee a better prediction for the band gap. In spite of the above observations, our work shows that for the forty one semiconductors considered, the mBJLDA potential represents an important improvement as compared to the results obtained from the previous version of the code. This procedure, all together, only mimics many-body results. But the mimic is not so bad for reason that do not seem to have theoretical foundation.

In a further work [40] we have compared the performance of some different methods in the literature to predict the band gap of semiconductors. We find that the GW approximation (GWA), a theoretical well founded method, gives the best result. The empirical potential in spite of the several problem and shortcomes described in this work gives predictions that compare acceptable well. Another factor that might be considered is the computational cost. GWA has a higher computational cost. It should be mentioned that this factor looses importance as new computer facilities spread all over the world as it is happening nowadays. We find, nevertheless, that there are still important issues to 
be fixed in DFT before we consider the proper prediction of the band gap value of semiconductors, a closed problem.

[1] P. Blaha, K. Schwars, G.K.H. Madsen, D. Kvasnicka, and J. Luitz, WIEN2K:Full Potential-Linearized Augmented Plane waves and Local Orbital Programs for Calculating Crystal Properties, edited by K. Schwars, Vienna University of Technology, Austria, (2001).

[2] F. Tran and P. Blaha, Phys. Rev. Lett. 102, 226401 (2009)

[3] G. K. H. Madsen, P. Blaha, K. Schwarz, E. Sjstedt, and L. ordstrm, Phys. Rev. B 64, 195134 (2001).

[4] P. Hohenberg and W. Khon, Phys. Rev. 136, B864 (1964).

[5] W. Khon and L.J. Sham, Phys. Rev. 140, A1133 (1965).

[6] J.P. Perdew and Y. Wang, Phys. Rev. B 45, 13244 (1992).

[7] J.P. Perdew, S. Kurth, J. Zupan, and P. Blaha, Phys. Rev. Lett. 82, 2544 (1999).

[8] A.D. Becke and E.R. Johnson, J. Chem. 124221101 (2006).

[9] A. D. Becke and M.R. Roussel, Phys. Rev. A 39, 3761 (1989).

[10] Murnaghan F.D., Am. J. Math. 59, 235 (1937).

[11] F. Birch, Phys. Rev. 71809 (1947)

[12] D. M. Teter, G. V. Gibbs, M. B. Boisen, Jr., D. C. Allan, M. P. Teter, J. Chem. Phys. 123, 174101 (1995).

[13] M. L. Cohen, Phys. Rev. B, 32, 798 (1985).

[14] P.E. Van Camp, V.E. Van Doren, and J. T. Devreese, Phys. Rev. B 38, 12675 (1988).

[15] Yingwei Fei, American Mineralogist 84, 272 (1999).

[16] R.A. Miller and Charles S. Smith, J. of Phys. and Chem. of Sol. 25, 1279 (1964).

[17] V. Fiorentini, Phys. Rev. B 46, 2086 (1992).

[18] J. V. Aleksandrov, A. F. Goncharov, S. M. Stishov, and E. Y. Yakovenko, JETP Lett. 50, 127 (1989).

[19] S-H. Wei and A. Zunger, Phys. Rev. B 60, 5404 (1999).

[20] D. H. Yean and J. R. Riter, J. Phys. Chem. Solids, 32653 (1971).

[21] A. Zaoui, and F. El Haj Hassan, Superlattices and Microstructures, 32, 91 (2002).

[22] H. J. McSkimin, A. Jayaraman. P. Andreatch, J. Appl. Phys. 38, 2362 (1967).

[23] S. Adachi, Handbook on Physical Properties of Semiconductors, Vols. I, II and III. Kluwer Academic Publishers (2004).

[24] O. Madelung, Data in Sciene and Technology, Semiconductors Group IV Elements and II-V Compounds. Ed. SpringerVerlag (1991).

[25] O. Madelung, Semiconductors: Data Handbook CD-ROM. Ed. Springer-Verlag (2003).

[26] J. N. Plendl and L. C. Mansur, Appl. Opt. 11, 1194 (1972).

[27] A. Taylor and R. M. Jones, in Silicon Carbide, A High Temperature Semiconductor, edited by J. R. O'Connor and J. Smiltens (Pergamon, London, 1960), p. 1470.

[28] J.L. Staudenmann, R. D. Horning, R. D. Knox, D. K. Arch, and J. L. Schmit, Appl. Phys. Lett. 48, 994 (1986).

[29] A. Bouhemadou, R. Khenata, F. Zegrar, M. Sahnoun, H. Baltache and A.H. Reshak, Comp. Mat. Sc., 2, 263 (2006).

[30] W. M. Vim and R. J. Paff, J. Appl. Phys. 45, 1456 (1974).

[31] R. R. Reeber, J. Appl. Phys. 41, 5063 (1970).

[32] N. Wang, M. Rohlfing, P. Krüger, and J. Pollmann, Phys.Rev. B 67, 115111 (2003).

[33] S. Antoci and L. Mihich, Phys.Rev. B 21, 799 (1980).

[34] D. Wolverson, D. M. Bird C. Bradford, K. A. Prior, and B. C. Cavenett, Phys. Rev. B, 64, 113203 (2001).

[35] K. Watanabe, M. Th. Litz, M. Korn, W. Ossau, A. Waag et al. J. Appl. Phys. 81, 451 (1997).

[36] M. Feneberg, J. Daubler, K. Thonke, and R. Sauer. Phys. Rev. B, 77, 245207 (2008).

[37] H.Morkoc, Handbook of Nitride Semiconductors and Devices, Ed. Wiley-VCH (2008).

[38] D.M. Roessler and W.C. Walker, J. Phys. Chem. Solids, 28, 1507 (1967).

[39] G. Ramírez-Flores, H. Navarro-Contreras, A. Lastras-Martínez, R. C. Powell and J. E. Greene, Phys. Rev. B 50, 8433 (1994).

[40] J. A. Camargo-Martínez and R. Baquero, arXiv:1208.205\%v1 [cond-mat.str-el] 